\title{
Gut microbiota in Immunoglobulin A Nephropathy: a Malaysian Perspective
}

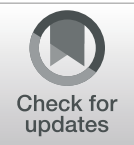

Sugurmar Agni Nhirmal Kumar ${ }^{*}$, Rozita Mohd', Shamsul Azhar Shah², Hui-min Neoh ${ }^{3}$ and Rizna Abdul Cader ${ }^{1,4}$

\begin{abstract}
Introduction: The alteration of the gut microbiome in the gut-kidney axis has been associated with a proinflammatory state and chronic kidney disease (CKD). A small-scaled Italian study has shown an association between the gut microbiome and Immunoglobulin A Nephropathy (IgAN). However, there is no data on gut microbiota in IgAN in the Asian population. This study compares the gut microbial abundance and diversity between healthy volunteers and Malaysian IgAN cohort.

Methods: A comparative cross-sectional study was conducted involving biopsy-proven IgAN patients in clinical remission with matched controls in a Malaysian tertiary centre. Demographic data, routine blood and urine results were recorded. Stool samples were collected and their DNA was extracted by $16 \mathrm{~S}$ rRNA gene sequencing to profile their gut microbiota.

Results: Thirty-six IgAN patients (13 male; 23 female) with the mean age of $45.5 \pm 13.4$ years and median estimated glomerular filtration rate (eGFR) of $79.0(62.1-92.2) \mathrm{mls} / \mathrm{min} / 1.73 \mathrm{~m}^{2}$ with median remission of 7 years were analysed and compared with 12 healthy controls ( 4 male; 8 female) with the mean age of $46.5 \pm 13.5$ years and eGFR of 86.5 (74.2-93.7) $\mathrm{mls} / \mathrm{min} / 1.73 \mathrm{~m}^{2}$. Other demographic and laboratory parameters such as gender, ethnicity, body mass index (BMI), haemoglobin, serum urea and serum albumin were comparable between the two groups. There were no significant differences seen in the Operational Taxonomic Unit (OTU) and alpha diversity (Shannon index) between IgAN and healthy controls. Alpha diversity increased with increasing CKD stage ( $p=0.025)$. Firmicutes/ Bacteroidetes (F/B) ratio was low in both IgAN and healthy cohort. Fusobacteria phylum was significantly increased $(p=0.005)$ whereas Euryarchaoeota phylum was reduced $(p=0.016)$ in the IgAN group as compared to the control cohort.
\end{abstract}

Conclusion: Although we found no differences in OTU and alpha diversity between IgAN in remission and control cohort, there were some differences between the two groups at phylum level.

Keywords: Gut microbiota, IgA nephropathy, Alpha diversity, Chronic kidney disease, Microbiome, Dysbiosis, Firmicutes/Bacteroidetes ratio

\footnotetext{
* Correspondence: agnishevchenko@gmail.com

'Department of Medicine, Universiti Kebangsaan Malaysia Medical Centre,

Jalan Yaacob Latif, Bandar Tun Razak, 56000 Kuala Lumpur, Malaysia

Full list of author information is available at the end of the article
}

(c) The Author(s). 2021 Open Access This article is licensed under a Creative Commons Attribution 4.0 International License, which permits use, sharing, adaptation, distribution and reproduction in any medium or format, as long as you give appropriate credit to the original author(s) and the source, provide a link to the Creative Commons licence, and indicate if changes were made. The images or other third party material in this article are included in the article's Creative Commons licence, unless indicated otherwise in a credit line to the material. If material is not included in the article's Creative Commons licence and your intended use is not permitted by statutory regulation or exceeds the permitted use, you will need to obtain permission directly from the copyright holder. To view a copy of this licence, visit http://creativecommons.org/licenses/by/4.0/ The Creative Commons Public Domain Dedication waiver (http://creativecommons.org/publicdomain/zero/1.0/) applies to the data made available in this article, unless otherwise stated in a credit line to the data. 


\section{Introduction}

Immunoglobulin A Nephropathy (IgAN) also known as synpharingitic nephritis is the most prevalent form of primary glomerulonephritis (GN) worldwide [1]. This autoimmune disease tends to affect the youth and accounts for $23 \%$ of $\mathrm{GN}$ in Malaysia [2]. In certain European countries, the reported prevalence was $>30 \%$ [1]. The depicted local prevalence is underestimated as a histopathological examination is not done comprehensively for all suspected cases and the existence of subclinical or indolent IgAN [3].

IgAN is a disease of abnormal Immunoglobulin A (IgA); it forms immune complexes that deposit into the mesangium and capillary walls causing glomerular injury and glomerulonephritis that manifest as nephritic syndrome. Abnormal IgA is due to defective glycosylation process caused by galactose deficiency [4]. Accumulation of abnormal IgA will bind to antiglycan Immunoglobulin G and form immune complexes. The exact pathogenesis is still ambiguous, but numerous studies postulate the multihit theory and the role of genes as possible causes $[5,6]$.

IgA is mainly found in the mucosal layers of the gut, genitourinary, respiratory tract, saliva, breast milk and tears [7]. Contemporary studies demonstrate that the gut is not only responsible for most of IgA production but also utilises IgA for maintaining gut mucosal colonisation [8]. There are more than a 1000 different species identified in the human gut and this community is termed as gut microbiome [9]. These vast arrays of microbiomes are in a perpetual symbiotic relationship between one another and the host, providing trophic and protective functions [10] including the development of the metabolic system, maturation of the intestinal immune system and the catering of essential nutrients [11, 12]. However, gut microbiota is heavily influenced by diet, environmental factors and socioeconomics, along with host genotype and genetic predisposition [13, 14].

Dysbiosis of the gut microbiome is increasingly recognised to be associated with various medical conditions including chronic kidney disease (CKD) [15-18]. Vaziri et al. demonstrate that CKD was associated with lower diversity and richness of gut microbiota and attributed these to the elevated urea in CKD [18].

Firmicutes and Bacteroidetes are the major phyla representing the gut microbiome $[19,20]$. Novel researches have emphasized on the Firmicutes to Bacteroidetes ratio - F/B ratio, as a marker of gut dysbiosis. Data on F/B ratio in IgAN is scarce. Various studies of other autoimmune and metabolic diseases have shown inconclusive F/B ratio. Studies found reduced F/B ratio in type 1 diabetes mellitus and systemic lupus erythematous [21]. In contrast, the F/B ratio was increased in psoriasis, a different form of autoimmune disease [22]. Other studies involving obesity [23-25] and multiple sclerosis patients showed inconsistent $\mathrm{F} / \mathrm{B}$ ratio $[26,27]$. These calls for more research to delineate the $\mathrm{F} / \mathrm{B}$ ratio in IgAN. Additionally, the role of treating dysbiosis is also taking a forefront. In a mouse model study, reduced Lactobacillus was found in mice with lupus nephritis, and increasing Lactobacillus with caecal microbiota transplantation was associated with an improvement in both renal and overall survival [28].

The first data on gut microbiota in IgA was published in 2014 as De Angelis et al. demonstrated a lower density of microbes especially the Clostridium, Enterococcus, Lactobacillus, Bifidobacterium genera [29]. The F/B ratio, as well as Firmicutes and Proteobacteria phylum, were increased in the IgAN group compared to healthy controls. Another study on Henoch Schönlein Purpura found a low diversity and richness in the disease group and serum IgA levels exhibited a significant negative correlation with the genus Bifidobacterium [30].

Thus, we aim to study the association of IgAN and gut microbiota in Asians, bearing in mind that the frequency and severity of IgAN in Asian population is different from that of Caucasians [31]. Furthermore, Asians are genetically different with notable variations in geographical regions, climate, dietary habits and lifestyle compared to Europeans. Our primary objective was to compare gut microbiome profile of IgAN patients in remission with control group. Understanding the gut microbiota of Asian IgAN may someday facilitate targeted correction of dysbiosis and possibly improve renal and overall survival.

\section{Materials and methods}

\section{Study design and participants}

This was a comparative cross-sectional study involving biopsy-proven IgA nephropathy patients attending the nephrology clinic follow up at Universiti Kebangsaan Malaysia Medical Centre (UKMMC) from August 2019 to January 2020. We included patients with biopsy proven IgAN aged more than 18 years and in disease remission. We excluded patients with diabetes (type 1 and 2), liver diseases, autoimmune diseases, malignancies, gastrointestinal diseases, ischaemic heart diseases, pregnancy and patients with serum urea more than 20 $\mathrm{mmol} / \mathrm{L}$ or end-stage renal diseases. We also excluded patients who have travelled abroad or were receiving antibiotics, immunosuppressant or probiotics in the past 3 months prior to our study. The control cohort consists of mostly healthy individuals, patients with stable hypertension disease on single antihypertensive and corrected obstructive uropathy. They were recruited for comparison and matched by age, gender, ethnicity and Body Mass Index (BMI).

Before enrolment all subjects provided written informed consent. All subjects that fulfilled the study 
criteria and consented were asked to keep a dietary diary to ensure no marked change in their usual dietary practices. Biochemical and clinical tests including faecal sample collection were performed in accordance with relevant guidelines and regulations, including Good Clinical Practice guidelines. Demographic data and disease history were recorded. Routine blood tests including full blood count, renal profile and liver function test, urinalysis and urine protein-creatinine index (UPCI) were done. Estimated Glomerular Filtration Rate (eGFR) was calculated based on the CKD-EPI 2009 equation [32]. Subjects were briefed on effective stool collection with minimal contamination during a consultation and via a pamphlet.

The study was approved by UKMMC research and ethics committee (FF-2019-352) and registered with $\mathrm{Na}$ tional Medical Research Registry (NMRR-19-3331-51, 504). This study was jointly funded by a grant from the Malaysian National Kidney Foundation and Universiti Kebangsaan Malaysia.

\section{Stool sampling and DNA extraction}

Stool samples were taken at home and brought to the hospital within $6 \mathrm{~h}$ in cold storage.

Samples were stored at $-80^{\circ} \mathrm{C}$ in the laboratory. DNA extraction was performed using GeneAll Exgene ${ }^{\mathrm{Tm}}$ Stool DNA kit (Cambio Ltd., Cambridge, England) as per manufacturer protocol. Concentration and quality of DNA extracts were monitored using Nanodrop Spectrophotometer (Nanodrop Technologies, Wilmington, Delaware).

\section{Gene sequencing - 16S rRNA analysis}

Following DNA extraction, 16S rRNA gene fragments were amplified from the extracted DNA. The genespecific sequences used in the protocol targets the $16 \mathrm{~s}$ rDNA V3 and V4 region as published in the literature [33]. Illumina adapter overhang nucleotide sequences were added to the gene-specific sequences. The fulllength primer sequences, using standard International Union of Pure and Applied Chemistry (IUPAC) nucleotide nomenclature, to follow the protocol targeting this region were:

16S Amplicon PCR Forward Primer $=5^{\prime}$

TCGTCGGCAGCGTCAGATGTGTATAAGAGA

CAGCCTACGGGNGGCWGCAG

16S Amplicon PCR Reverse Primer $=5^{\prime}$ GTCTCG TGGGCTCGGAGATGTGTATAAGAGACAGGACTAC HVGGGTATCTAATCC

Forward overhang: 5'

TCGTCGGCAGCGTCAGATGTGTATAAGAGACAG[locus-specific sequence]

Reverse overhang: $5^{\prime}$
GTCTCGTGGGCTCGGAGATGTGTATAAGAG

$A C A G$ - [locus-specific sequence]

Reads from the sequencing were sorted by bcl2fastq 2 software using unique barcodes. The barcode, linker, and primer sequences were then removed from the original sequencing reads. The merged reads containing two or more ambiguous nucleotides, those with a lowquality score (average score $<20$ ), or reads shorter than 300 Base pair, were filtered out. Potential chimeric sequences were detected using the ChimeraSlayer r20110519.

The pre-processed reads from each sample were used to calculate the number of OTUs which was determined by clustering the sequences from each sample using a 97\% sequence identity cut-off using Quantitative Insights Into Microbial Ecology (QIIME) software (v.1.8.0).

Taxonomic abundance was counted with RDP Classifier v2.11 using a confidence threshold of 0.8 derived from the pre-processed reads for each sample and NCBI Blast v2.2.28 following clustering by CD-HIT v4.6 using a $99 \%$ sequence identity with $80 \%$ read coverage cut-off $[34,35]$. The microbial composition was normalized by dividing the value calculated from the taxonomy abundance count with the number of pre-processed reads for each sample. To measure the alpha diversity of each sample, the OTUs were analysed using the Shannon index, $H^{\prime}=-\sum_{i=1}^{S}\left(p_{i} \ln \left(p_{i}\right)\right)$.

\section{Statistical analysis}

All data presented in this study were analysed using SPSS software version 21 (IBM Inc., Chicago, IL, USA). The sample size was calculated based on the only available study describing gut microbiota and IgAN at the time, De Angelis et al. [29]. We required 8 experimental subjects and 8 control subjects to be able to reject the null hypothesis that the population means of the experimental and control groups are equal with probability (power) 0.8. The Type I error probability associated with this test of this null hypothesis is 0.05 . We recruited more considering the possibility of dropouts and sampling error.

The rationale behind 3:1 ratio was due to a myriad of variables that may influence the gut microbiome. Statistical significance can be achieved by conventional 1:1 ratio but increasing the number participants in the disease group will reduce the weight of these unaccounted variables as well as reduce selection bias.

All data were tested for normality. Next, normally distributed data were analysed with mean and standard deviation while skewed distributed data were analysed with median and interquartile range (25-75\%). Independent t-test and Mann Whitney test were used to compare the variables. In order to adjust for false discovery rate, we 
Table 1 Demographic and laboratory data in IgAN patients and healthy controls

\begin{tabular}{|c|c|c|c|}
\hline & IgAN cohort $(n=36)$ & Healthy cohort $(n=12)$ & $P$ value \\
\hline & Mean \pm SD/ Median (IQR) & Mean \pm SD/ Median (IQR) & \\
\hline Age (years) & $45.5 \pm 13.4$ & $46.5 \pm 13.5$ & 0.814 \\
\hline \multicolumn{4}{|l|}{ Gender [n, (\%)] } \\
\hline Male & $13(36 \%)$ & $4(33 \%)$ & \multirow[t]{2}{*}{0.865} \\
\hline Female & $23(64 \%)$ & $8(67 \%)$ & \\
\hline \multicolumn{4}{|l|}{ Race $[n,(\%)]$} \\
\hline Malay & $17(47.2 \%)$ & $6(50 \%)$ & \multirow[t]{2}{*}{0.871} \\
\hline Chinese & 19 (52.8\%) & $6(50 \%)$ & \\
\hline BMI $\left(\mathrm{kg} / \mathrm{m}^{2}\right)$ & $24.56 \pm 2.79$ & $23.62 \pm 3.03$ & 0.326 \\
\hline Haemoglobin (g/dL) & $13.43 \pm 1.56$ & $13.18 \pm 1.54$ & 0.707 \\
\hline Urea $(\mathrm{mmol} / \mathrm{L})$ & $4.7(4.2-5.7)$ & $4.0(3.4-5.0)$ & 0.072 \\
\hline Creatinine $(\mu \mathrm{mol} / \mathrm{L})$ & $83.5(62.3-116.5)$ & $69.5(59.3-84.3)$ & 0.14 \\
\hline eGFR (mls/min/1.73m²) & $79.0(62.1-92.2)$ & 86.5 (74.3-93.8) & 0.248 \\
\hline Albumin (g/L) & $41.39 \pm 3.36$ & $42.33 \pm 1.97$ & 0.245 \\
\hline UPCI (g/mmol creatinine) & $0.050(0.023-0.128)$ & $0.01(0.01-0.01)$ & $<0.001$ \\
\hline
\end{tabular}

BMI Body Mass Index, eGFR estimated Glomerular Filtration Rate based on CKD EPI 2009

UPCI Urine Protein-Creatinine Index

used the Bonferroni multiple comparison correction. Our adjusted $p$ value / $\mathrm{q}$ value of 0.05 was considered statistically significant.

\section{Results}

\section{Subject profiles and characteristics}

We recruited a total of 36 IgAN patients with 12 controls. Both groups were comparable in their demographics parameters including age, gender, ethnic distribution, as well as BMI and eGFR. Biochemical parameters were also comparable except for UPCI that was higher in the IgAN group and summarised in Table 1. The IgAN subjects were in clinical remission for at least 1 year with a median duration of remission being 7 years (IQR 4-13). Majority of our patients had only 1 relapse and all treated as per KDIGO guideline with antiproteinuric agents, namely Angiotensin-Converting Enzyme inhibitor (ACE-i) or Angiotensin II Receptor Blocker (ARB) [36]. All 36 patients (100\%) in our IgAN cohort were treated with either ACE-i or ARB and 23 patients (63.9\%) were on fish oil therapy. 7 out of 12 (58\%) control patients were on ACE-i/ARB for their hypertension. All these patients have good hypertension control on ACE-i/ARB.

\section{Characterisation of intestinal microbiome}

The number of reads in all 48 subjects by $16 \mathrm{~S}$ rRNA V3 and V4 amplicon sequencing was $3,797,148$. This final read number was obtained post trimming and quality control. The median for the number of reads for disease cohort was 87,125 $(54272-99,417)$ as shown in Table 2.

There were no significant differences in OTUs or alpha diversity measured in the Shannon index between IgAN and controls. Further analysis indicated no relationship between the number of OTUs and Shannon index with ethnicity, gender or BMI. However, the IgAN cohort had significant association of alpha diversity with eGFR $<60 \mathrm{mls} / \mathrm{min} / 1.73 \mathrm{~m}^{2}, p=0.025$. The alpha diversity increased with the reduction of eGFR. These findings were not found in the control cohort.

A total of 24 phyla, 48 classes and 685 genera were identified. Six major phyla dominated the composition of the gut microbiota - Bacteroidetes, Firmicutes, Proteobacteria and to a lesser extent Verrucomicrobia, Actinobacteria and Fusobacteria. These phyla are shown in each individual subject in Fig. 1. Figure 2 demonstrates the composition of these phyla between the two cohorts. IgAN cohort had an increased abundance in Proteobacteria, Fusobacteria and Actinobacteria but reduced

Table 2 Gut microbiome analysis between the two groups

\begin{tabular}{llll}
\hline & IgAN cohort $(\boldsymbol{n}=\mathbf{3 6})$ & Control cohort $(\boldsymbol{n}=\mathbf{1 2})$ & $P$-value \\
& Mean \pm SD/ Median (IQR) & Mean \pm SD/ Median (IQR) & 0.849 \\
\hline Number of reads & $87,125(54273-99,417)$ & $84,188(74645-93,178)$ & 0.328 \\
Number of OTUs & $10,080.64 \pm 3018.92$ & $11,015.33 \pm 2163.45$ & 0.739 \\
Shannon index & $4.986 \pm 0.508$ & $5.046 \pm 0.595$ & \\
\hline
\end{tabular}




\section{Major phyla in each individuals}

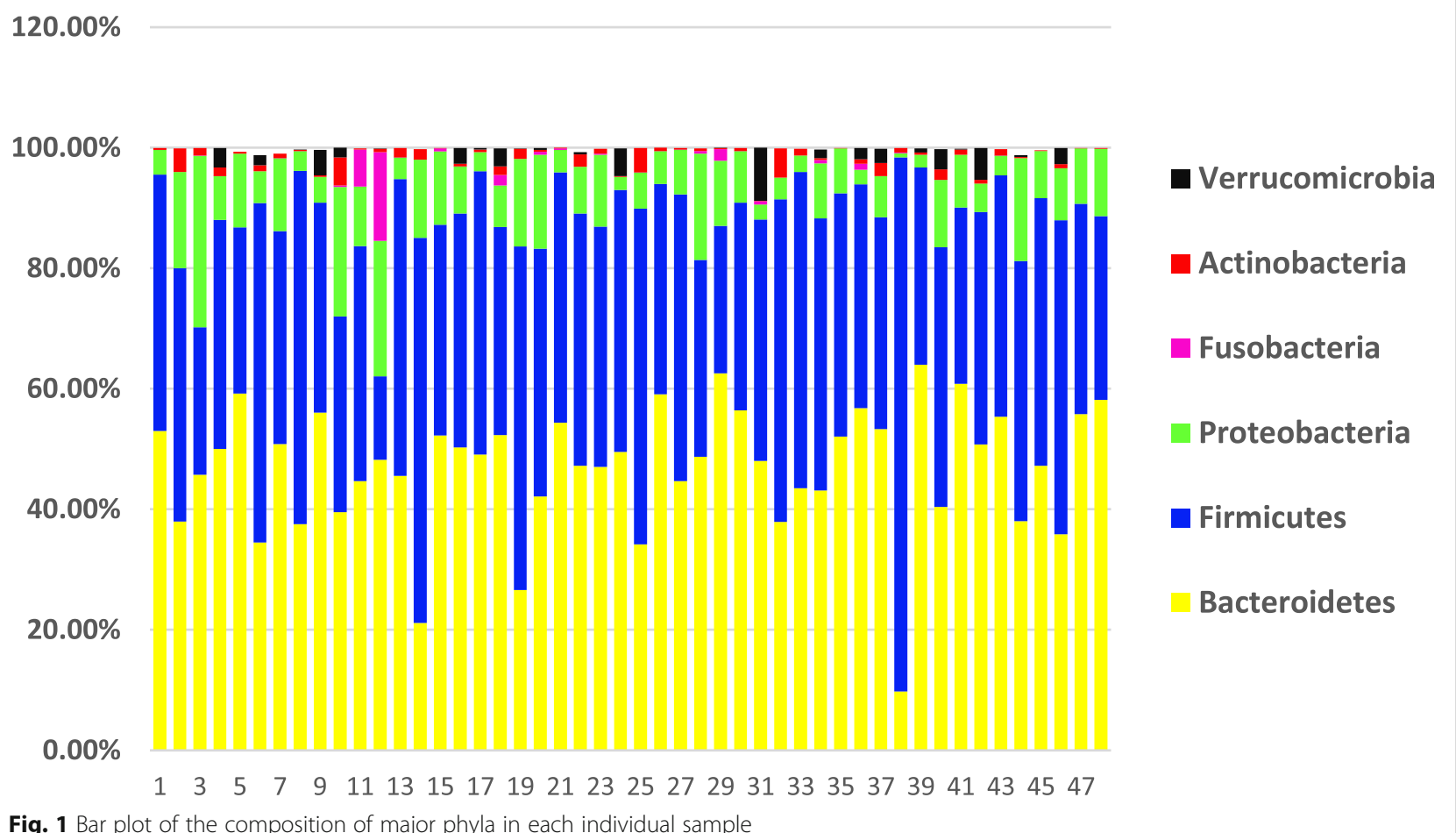

Firmicutes, Bacteriodetes and Verrucomicrobia when compared with controls as shown in Fig. 2. The Firmicutes / Bacteroidetes ratio (F/B ratio) was low in both our cohorts (0.87 in IgAN and 0.89 in healthy).

At the phylum level, the proportion of Fusobacteria was higher in the IgAN cohort $(p=0.005)$ whereas Euryarchaoeota was lower in the IgAN cohort $(p=0.016)$ and this is shown in Fig. 3a.

At the class level, Methanobacteria was significantly reduced in the IgAN cohort $(p=0.031)$ whereas Fusobacteriia $(\mathrm{p}=0.005)$ and Epsilonproteobacteria ( $p=$
0.018) were increased in the IgAN cohort and shown in Fig. 3b.

Analysing at the genus level, although Streptococcus was increased in IgAN group compared to the controls, it was not significant $(p=0.432)$. Clostridium, Bacillus and Lactobacillus, the mainstay microorganisms in the gut, were reduced in the IgAN group but these too did not reach statistical significance.

On subanalysis of the IgAN cohort, we found that patients with eFGR less than $60 \mathrm{mls} / \mathrm{min} / 1.73 \mathrm{~m}^{2}$ had an increase in the phyla Lentisphaerae ( $p=$

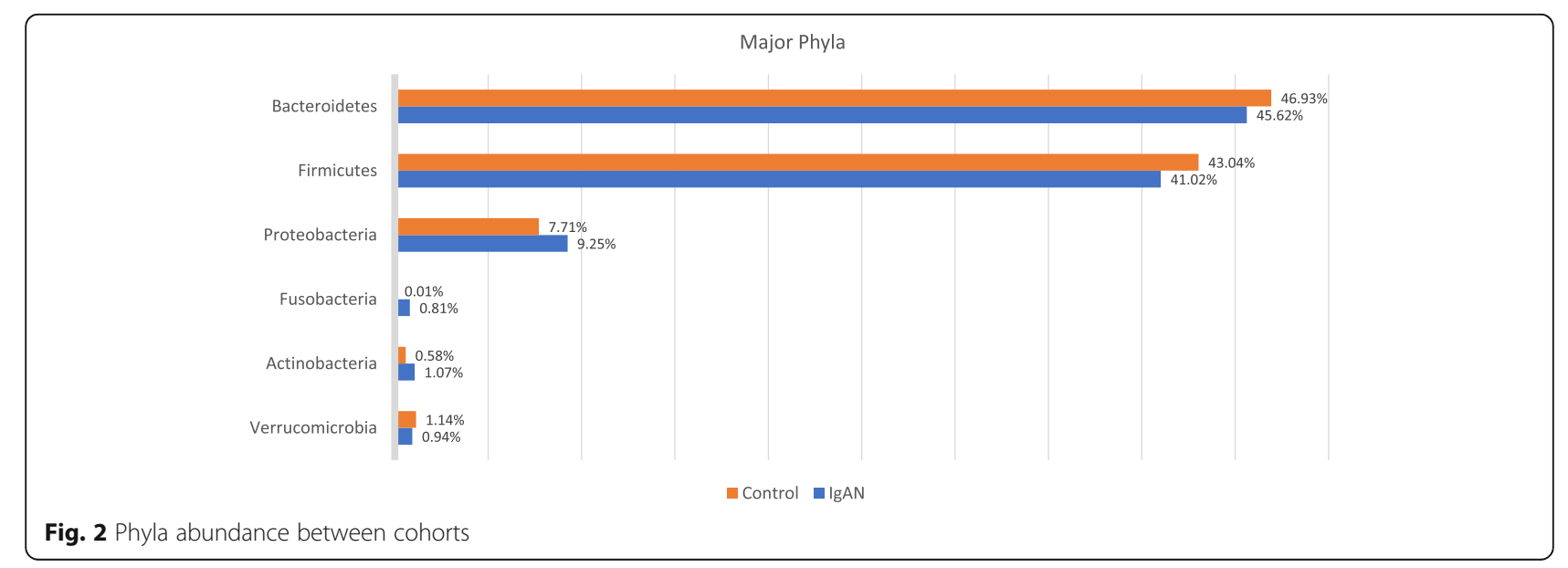



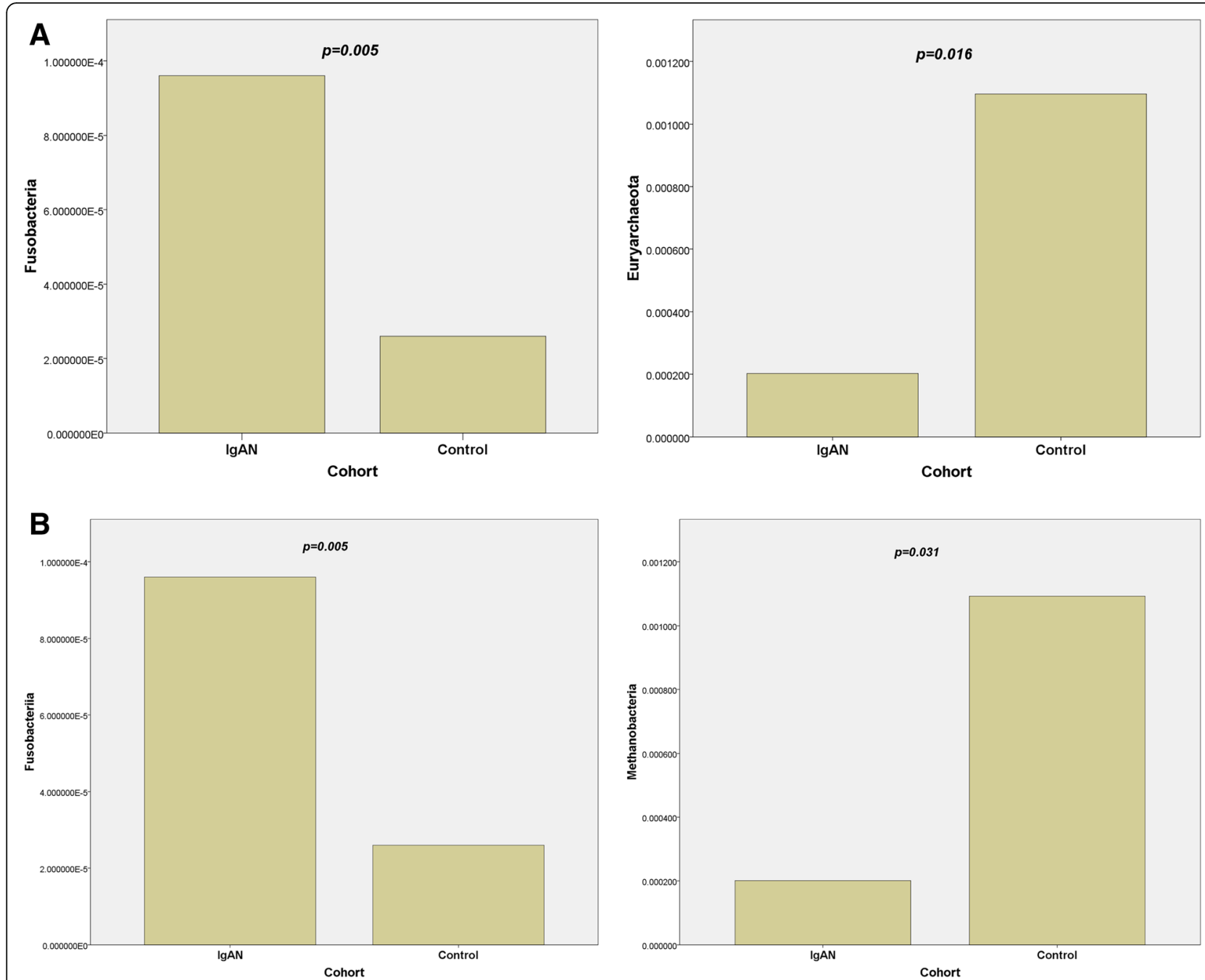

Fig. 3 Differences in gut microbiota between IgAN and control cohort. a At phylum level (b) At class level

$0.001)$ and Synergistetes $(p=0.002)$ and displayed in Fig. 4.

\section{Discussion}

Malaysia has a multiracial population and the prevalence of IgAN is $23 \%$ with a slight preponderance towards female (1.44:1) [2]. This is lower compared to our neighbouring country, Singapore, where IgAN was reported as high as $43 \%$ [37]. However, Malaysian data maybe under-representative of the true incidence due to different screening methods and renal biopsy policies practised.

IgAN poses a substantial risk of progression to ESRD, up to $40 \%$ in two decades especially in Asians $[31,38]$. Blood pressure reduction and treatment with reninangiotensin-aldosterone system blockers or immunosuppressant [39] have been advocated to slow the CKD progression although the use of the latter remains controversial with conflicting evidence [40]. In our centre, immunosuppressive therapies are used for patients with heavy proteinuria as Asian studies have shown promising results with steroids [41]. All of our IgAN patients were on either ACE-I or ARB while 64\% were on fish oil. 7 out of 12 from the control cohort were on ACE-i/ARB for their hypertension, which was well controlled. The actions of common medications on the gut microbiota cannot be discounted. ACE-i has shown in animal models to improve gut dysbiosis by reducing intestinal permeability, decreasing fibrosis and improving villi length [42]. However, at present, the available data records no obvious changes at phyla level.

Fish oil, rich in polyunsaturated fatty acids, is still widely used as it poses low risk and has a potentially beneficial cardiovascular effects [43]. Fish oil has been studied elaborately to determine its effect on gut microbiota. Some studies have shown no differences in the phyla level $[44,45]$ while others have shown an increase in Firmicutes and decrease in Bacteroidetes [46-48]. 

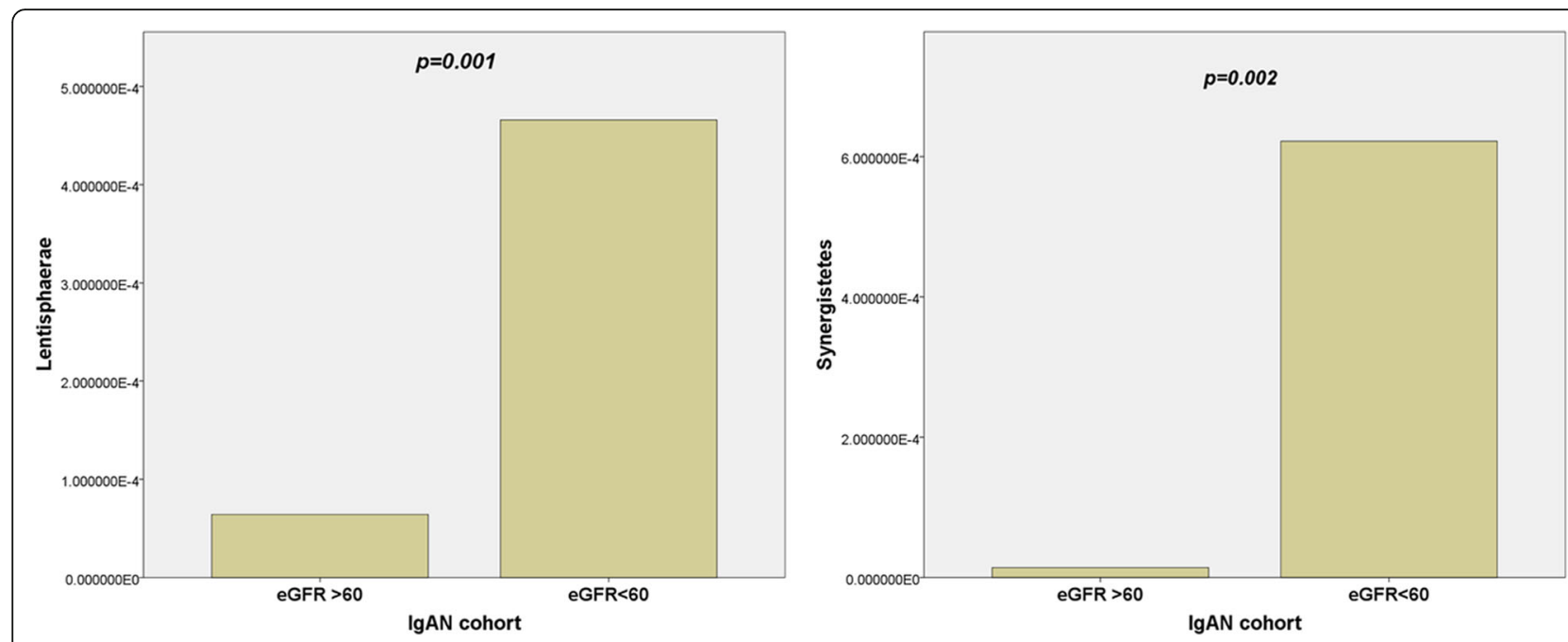

Fig. 4 eGFR influencing abundance at the phylum level

However, none have shown any changes in the Fusobacteria phylum. Both our cohorts did not show significant changes in the Firmicutes and Bacteroidetes phyla.

We decided not to include patients with newly diagnosed who are in active disease or relapsed disease due to several factors. Firstly, our aim is to prove that patients with the disease and in remission are distinguishable from the control group. This theory is needed to confirm that there is fundamental alteration in gut microbiota that plays a role in the pathogenesis of the disease. Secondly, patients with active disease have more variables that results in dysbiosis, - some are clinically ill with ongoing acute kidney injury, while others have hypoalbuminemia which may cause oedema including in the gut. On top of that, in our local practice, patients with active disease are considered for immunosuppressants. All these factors are proven to alter gut microbiota.

Our IgAN cohort was noted to have significantly higher UPCI compared to controls. This is largely due to the fact that these patients have progressed to CKD and have persistent stable proteinuria due to glomerulosclerosis. The eGFR in control group were slightly low as there were patients with CKD stage 3 due to corrected obstructive uropathy. These patients were including to offset the differences of eGFR in between both groups, as CKD severity has already been reported to influence gut microbiota [18].

We discovered no significant difference in OTU richness, gut microbial diversity in IgAN compared to controls. We perceive this could be because all our IgAN patients were in disease remission and not on any immunosuppressive drugs, thus resembling the healthy population. In addition, the assessment of alpha diversity using the Shannon index may have some limitation as shown in other studies [25, 49]. Bacteroidetes, Firmicutes, Proteobacteria, Verrucomicrobia, Actinobacteria and Fusobacteria constituted $>98 \%$ of total abundance and it is similar to other studies worldwide $[9,29,50$, 51]. Bacteroidetes and Firmicutes monopolise the overall microbiota. Bacteroidetes is represented mainly by Bacteroides and Prevotella whereas the Firmicutes consists of mainly the genus Clostridium.

The calculated F/B ratio was low in general and reduced further in IgAN cohort. This was due to a decrease in abundance of Clostridia. Studies in the European continent have reported a high F/B ratio $>1$ $[29,50,51]$ whereas the Asian and African continents reported otherwise, $<1[52,53]$. These differences in gut microbiota profile are likely due to the variation in the diet in which more animal-based protein and fat observed in the Europe diet whereas Asian and African diet is traditionally more plant-based [54]. Significantly greater alpha diversity, increase in OTU and specifically, Bacteroidetes phylum were seen with vegans compared to omnivores [55]. These changes in the composition of the microbiome are often due to differences in the amount of directly consumed bacteria, variation in transit time through the intestinal tract, changes in gut $\mathrm{pH}$ and regulation of host immunity [56].

The crucial outcome of our study was the significant increase of Fusobacteria in the IgAN cohort. Fusobacteria has the ability to invade colonic epithelial cells [57]. As such, studies have been conducted to prove its association with inflammatory bowel disease and colon cancer [58]. Fusobacteria touted to play a role as an "alpha-bug" which has a virulent capacity to spur remodelling of the entire community, cultivating a proinflammatory and pro-carcinogenic response [59]. Could this be the intermediary for abnormal mucosal immunity 
and gut inflammation that initiates the multi-hit pathogenesis of IgAN?

The Euryarchaoeota was significantly lower in the IgAN cohort. This microorganism falls under the domain of Archaea, an obligate anaerobe which is separate from bacteria. They are known to produce methane as a metabolic by-product in hypoxic conditions [60]. There are links between this organism and obesity, constipation and oral cavity infections [61] but we have yet to put this incidental finding in its place.

A unique finding to our study was the increase in alpha diversity with the worsening of CKD. This finding is in contradiction to the general understanding that gut microbial diversity reduces with increasing severity of CKD. Our understanding of this phenomenon is that gut microbiota is predominantly affected by serum urea and toxins such as p-cresol rather than creatinine level [62]. We did not encounter this as there were no significant differences in the urea level between the two groups and the urea level was relatively low in our IgAN cohort. We also report an increase in Lentisphaerae $(p=0.001)$ and Synergistetes $(p=$ 0.002 ) in patients with eGFR less than $60 \mathrm{mls} / \mathrm{min} / 1.73 \mathrm{~m}^{2}$. As the relative abundance of these phyla is minute, very few studies have shown their role in our gut ecology.

As IgAN patients often worsen after suffering an upper respiratory tract infection, Streptococcal antigens have been implicated with the deposition of IgA in renal tissue [63]. Our findings with regards to Streptococcus compared between the two cohorts showed no difference. This may echo with the consensus that the role of tonsillectomy for the purpose of reduction in Streptococcus exposure may be limited [36]. Having said that, the role of Streptococcus as a gut coloniser may differ in tonsil infection. Moreover, our Streptococcus could have been indifferent in both cohorts as our patients were mostly in remission.

Notable discrepancies in our study as compared to that of De Angelis' et al. [29] would be that our F/B ratio was reduced in the IgAN group whereas the Italians reported an increase. Our Firmicutes abundance was reduced in IgAN cohort while Fusobacteria and Actinobacteria increased in IgAN cohort contrasting the results of the Italian study. Previous studies have also documented a reduction in Firmicutes in disease cohorts $[62,64,65]$. Our reduction in Firmicutes is mainly due to reduction in the genus Clostridium. Clostridium is responsible for the metabolism of carbohydrate to various short-chain fatty acids (SCFA). SCFAs are needed to maintain the intestinal barrier and its reduction will trigger inflammation [66]. We believe that as our IgAN cohort was in prolonged remission, we were unable to achieve statistical significance in the reduction of phyla Firmicutes and genus Clostridium.

Recently, a new study out of Hunan, China - looking into faecal microbiota characteristics of active IgAN and healthy control, has demonstrated similar findings to ours [67]. They have recorded a reduction in all alpha diversity indexes except Shannon index. Using the Shannon index as the only measurement for alpha diversity has its limitations [68]. In fact, there are still ongoing arguments to justify the best method to evaluate richness diversity, evenness, differentiation and abundanceweighted diversity [69]. Hu et al. also noted a significant increase in the phyla Fusobacteria in their active disease cohort [67]. However, their F/B ratio was higher in the diseased group, concurring with De Angelis et al.

Some notable weaknesses from our study would be our design - cross-sectional study and its sample size. Although the sample size was calculated with type 1 error of 0.05 and power of $80 \%$, a larger-scale study will be needed to confirm these results as gut microbiome has high dimensionality and variability. Another major limitation was the lack of other indexes to evaluate Alpha diversity and Beta diversity. This is mainly due to technical issues we were facing during the time of COVID-19 pandemic. Even then we believe this study is still relevant as it is the only study on IgAN patients who were in remission. Remission state reduces many other confounding variables that may alter the gut microbiome such as patients being unwell during acute illness, hypoalbuminemia, acute kidney injury, as well as a sudden change in medication and diet. Moreover, we contribute to the database of gut microbiota profile for the ASEAN region.

\section{Conclusion}

The richness and abundance of OTU and alpha diversity are unaltered between IgAN patients in remission and controls. Nonetheless, there were significant differences in taxonomic profiling even at the phylum level. Thus, these results might be relevant to understand the basis of microbiota perturbation in the development of IgAN in the Malaysian population. The phyla Fusobacteria may be a precursor to intestinal inflammation and autoimmunity in our population. Proving the role of gut dysbiosis as one of the pathogenesis to the development of this disease can facilitate a new population targeted screening, stratification and treatment approach.

\section{Supplementary Information}

The online version contains supplementary material available at https://doi. org/10.1186/s12882-021-02315-z.

\section{Additional file 1.}

Additional file 2.

Acknowledgements

The authors would like to thank the National Kidney Foundation (NKF), Malaysia for partly supporting us with a financial grant. We would also like to thank Arbaiyah Bain for her contribution. 


\section{Authors' contributions}

Dr. ANKS: Acquisition of data, conducting the study, data analysis, interpretation of data, statistical analysis and drafting of manuscript. Dr. RC: Study concept, administrative and technical support, data analysis, interpretation of data, critical revision of the manuscript for important intellectual content. Assoc. Professor RM: Study supervision, critical revision of the manuscript for important intellectual content. Professor SAS: Study design, statistical analysis. Assoc. Professor NHM: Study design, technical and material support. The author(s) read and approved the final manuscript.

\section{Funding}

This study was jointly funded by a grant from the Malaysian National Kidney Foundation and Universiti Kebangsaan Malaysia. No funders had a role in the design of the study, data collection, analysis and data interpretation, or writing of the manuscript.

\section{Availability of data and materials}

The datasets generated during and analysed during the current study is available in the Zenodo repository. DOl: https://doi.org/10.5281/zenodo. 4575681

\section{Declarations}

\section{Ethics approval and consent to participate}

This study was approved by the Medical Ethics Committee of Universit Kebangsaan Malaysia Medical Centre, and it is in accordance with the Helsinki Declaration (IV adaptation). Written informed consent was obtained from all participants.

The authors declare that the research was conducted in the absence of any commercial or financial relationships that could be construed as a potential conflict of interest.

\section{Consent for publication}

Not applicable.

\section{Competing interests}

The authors declare that they have no competing interests.

\section{Author details}

'Department of Medicine, Universiti Kebangsaan Malaysia Medical Centre, Jalan Yaacob Latif, Bandar Tun Razak, 56000 Kuala Lumpur, Malaysia. 2Department of Community Health, Universiti Kebangsaan Malaysia Medical Centre, Kuala Lumpur, Malaysia. ${ }^{3}$ UKM Molecular Biology Institute, Kuala Lumpur, Malaysia. ${ }^{4}$ ParkCity Medical Centre, No.2, Jalan Intisari Perdana, Desa ParkCity, Kuala Lumpur 52200, Malaysia.

\section{Received: 1 November 2020 Accepted: 11 March 2021}

\section{Published online: 22 April 2021}

\section{References}

1. Schena FP, Nistor I. Epidemiology of IgA nephropathy: a global perspective. Semin Nephrol. 2018;38(5):435-42. https://doi.org/10.1016/j.semnephrol.201 8.05.013.

2. Rizna Abdul Cader ETZZ, Yahya R, Manocha AB. 6th Report of Malaysian registry of renal biopsy 2017. MRRB reports MSN. June 2019 ed. Kuala Lumpur: National Renal Registry; 2017. p. 22-8.

3. Suzuki K, Honda K, Tanabe K, Toma H, Nihei H, Yamaguchi Y. Incidence of latent mesangial IgA deposition in renal allograft donors in Japan. Kidney Int. 2003;63(6):2286-94. https://doi.org/10.1046/j.1523-1755.63.65.2.x.

4. Novak J, Julian BA, Mestecky J, Renfrow MB. Glycosylation of IgA1 and pathogenesis of IgA nephropathy. Semin Immunopathol. 2012;34(3):365-82. https://doi.org/10.1007/s00281-012-0306-z.

5. Suzuki H, Kiryluk K, Novak J, Moldoveanu Z, Herr AB, Renfrow MB, et al. The pathophysiology of IgA nephropathy. J Am Soc Nephrol. 2011;22(10):1795803. https://doi.org/10.1681/ASN.2011050464.

6. Feehally J, Farrall M, Boland A, Gale DP, Gut I, Heath S, et al. HLA has strongest association with IgA nephropathy in genome-wide analysis. J Am Soc Nephrol. 2010;21 (10):1791-7. https://doi.org/10.1681/ASN.2010010076.

7. Corthésy B. Multi-faceted functions of secretory lgA at mucosal surfaces. Front Immunol. 2013;4:185-5.
8. Donaldson GP, Ladinsky MS. Gut microbiota utilize immunoglobulin A for mucosal colonization. Science. 2018;360:795-800.

9. Harmsen HJ, de Goffau MC. The human Gut microbiota. Adv Exp Med Biol. 2016;902:95-108. https://doi.org/10.1007/978-3-319-31248-4 7.

10. Bourlioux P, Koletzko B, Guarner F, Braesco V. The intestine and its microflora are partners for the protection of the host: report on the Danone symposium "the intelligent intestine," held in Paris, June 14, 2002. Am J Clin Nutr. 2003;78(4):675-83. https://doi.org/10.1093/ajcn/78.4.675.

11. Neuman H, Debelius JW, Knight R, Koren O. Microbial endocrinology: the interplay between the microbiota and the endocrine system. FEMS Microbiol Rev. 2015;39(4):509-21. https://doi.org/10.1093/femsre/fuu010.

12. McDermott AJ, Huffnagle GB. The microbiome and regulation of mucosal immunity. Immunology. 2014;142(1):24-31. https://doi.org/10.1111/ imm.12231.

13. Chan CW, Wong RS, Law PT, Wong CL, Tsui SK, et al. Environmental factors associated with altered gut microbiota in children with eczema: a systematic review. Int J Mol Sci. 2016;17(7):1147.

14. Chong CW, Ahmad AF, Lim YA, Teh CS, Yap IK, et al. Effect of ethnicity and socioeconomic variation to the gut microbiota composition among preadolescent in Malaysia. Sci Rep. 2015;5(1):13338. https://doi.org/10.1038/ srep13338.

15. Kuhn KA, Pedraza I, Demoruelle MK. Mucosal immune responses to microbiota in the development of autoimmune disease. Rheum Dis Clin N Am. 2014;40(4):711-25. https://doi.org/10.1016/j.rdc.2014.07.013.

16. Qin J, Li Y, Cai Z, Li S, Zhu J, Zhang F, et al. A metagenome-wide association study of gut microbiota in type 2 diabetes. Nature. 2012; 490(7418):55-60. https://doi.org/10.1038/nature11450.

17. Hu J, Zhong X, Yan J, Zhou D, Qin D, Xiao X, et al. High-throughput sequencing analysis of intestinal flora changes in ESRD and CKD patients. BMC Nephrol. 2020;21(1):12. https://doi.org/10.1186/s12882-019-1668-4.

18. Vaziri ND, Wong J, Pahl M, Piceno YM, Yuan J, DeSantis TZ, et al. Chronic kidney disease alters intestinal microbial flora. Kidney Int. 2013;83(2):308-15. https://doi.org/10.1038/ki.2012.345.

19. Rinninella E, Raoul P, Cintoni M, Franceschi F, Miggiano GAD, Gasbarrini A et al. What is the healthy Gut microbiota composition? A changing ecosystem across age, environment, diet, and diseases. Microorganisms. 2019;7(1):14. https://doi.org/10.3390/microorganisms7010014.

20. Mariat D, Firmesse $\mathrm{O}$, Levenez F, Guimarăes VD, Sokol H, Doré J, et al. The Firmicutes/Bacteroidetes ratio of the human microbiota changes with age. BMC Microbiol. 2009:9(1):123. https://doi.org/10.1186/1471-2180-9-123.

21. Opazo MC, Ortega-Rocha EM, Coronado-Arrázola I, Bonifaz LC, Boudin H, Neunlist $M$, et al. Intestinal microbiota influences non-intestinal related autoimmune diseases. Front Microbiol. 2018;9:432-2. https://doi.org/10.33 89/fmicb.2018.00432

22. Masallat D, Moemen D, State A. Gut bacterial microbiota in psoriasis: a case control study. Afr J Microbiol Res. 2016;10:1337-43.

23. Koliada A, Syzenko G, Moseiko V, Budovska L, Puchkov K, Perederiy V, et al. Association between body mass index and Firmicutes/Bacteroidetes ratio in an adult Ukrainian population. BMC Microbiol. 2017;17(1):120. https://doi. org/10.1186/s12866-017-1027-1.

24. Kasai C, Sugimoto K, Moritani I, Tanaka J, Oya Y, Inoue H, et al. Comparison of the gut microbiota composition between obese and non-obese individuals in a Japanese population, as analyzed by terminal restriction fragment length polymorphism and next-generation sequencing. BMC Gastroenterol. 2015:15(1):100. https://doi.org/10.1186/s12876-015-0330-2.

25. Hu H-J, Park S-G, Jang HB, Choi M-G, Park K-H, Kang JH, et al. Obesity alters the microbial community profile in Korean adolescents. PLoS One. 2015; 10(7):e0134333. https://doi.org/10.1371/journal.pone.0134333.

26. Miyake S, Kim S, Suda W, Oshima K, Nakamura M, Matsuoka T, et al. Dysbiosis in the Gut microbiota of patients with multiple sclerosis, with a striking depletion of species belonging to clostridia XIVa and IV clusters. PLoS One. 2015;10(9):e0137429. https://doi.org/10.1371/journal.pone.0137429.

27. Tremlett H, Fadrosh DW, Faruqi AA, Hart J, Roalstad S, Graves J, et al. Gut microbiota composition and relapse risk in pediatric MS: a pilot study. J Neurol Sci. 2016;363:153-7. https://doi.org/10.1016/j.jns.2016.02.042.

28. Mu Q, Zhang H, Liao X, Lin K, Liu H, Edwards MR, et al. Control of lupus nephritis by changes of gut microbiota. Microbiome. 2017;5(1):73. https:// doi.org/10.1186/s40168-017-0300-8.

29. De Angelis M, Montemurno E, Piccolo M, Vannini L, Lauriero G, et al. Microbiota and Metabolome associated with immunoglobulin a nephropathy (IgAN). PLoS One. 2014;9(6):e99006. 
30. Wang X, Zhang L, Wang Y, Liu X, Zhang H, Liu Y, et al. Gut microbiota dysbiosis is associated with Henoch-Schönlein Purpura in children. Int Immunopharmacol. 2018;58:1-8. https://doi.org/10.1016/j.intimp.2018.03.003.

31. Barbour SJ, Cattran DC, Kim SJ, Levin A, Wald R, Hladunewich MA, et al. Individuals of Pacific Asian origin with IgA nephropathy have an increased risk of progression to end-stage renal disease. Kidney Int. 2013;84(5):101724. https://doi.org/10.1038/ki.2013.210.

32. Levey AS, Stevens LA, Schmid CH, Zhang YL, Castro AF 3rd, et al. A new equation to estimate glomerular filtration rate. Ann Intern Med. 2009;150(9): 604-12. https://doi.org/10.7326/0003-4819-150-9-200905050-00006.

33. Klindworth A, Pruesse E, Schweer T, Peplies J, Quast C, Horn M, et al. Evaluation of general 165 ribosomal RNA gene PCR primers for classical and next-generation sequencing-based diversity studies. Nucleic Acids Res. 2013; 41(1):e1. https://doi.org/10.1093/nar/gks808.

34. Wang Q, Garrity GM, Tiedje JM, Cole JR. Naive Bayesian classifier for rapid assignment of rRNA sequences into the new bacterial taxonomy. Appl Environ Microbiol. 2007;73(16):5261-7. https://doi.org/10.1128/AEM.00062-07.

35. Federhen S. The NCBI taxonomy database. Nucleic Acids Res. 2012;40(D1): D136-43. https://doi.org/10.1093/nar/gkr1178.

36. Radhakrishnan J, Cattran DC. The KDIGO practice guideline on glomerulonephritis: reading between the (quide)lines--application to the individual patient. Kidney Int. 2012;82(8):840-56. https://doi.org/10.1038/ki.2 012.280 .

37. Woo KT, Chan CM, Mooi CY, Choong H-L, Tan H-K, et al. The changing pattern of primary glomerulonephritis in Singapore and other countries over the past 3 decades. Clin Nephrol. 2010;74(11):372-83. https://doi.org/1 $0.5414 / C N P 74372$

38. Deng W, Tan X, Zhou Q, Ai Z, Liu W, Chen W, et al. Gender-related differences in clinicopathological characteristics and renal outcomes of Chinese patients with IgA nephropathy. BMC Nephrol. 2018;19(1):31. https:// doi.org/10.1186/s12882-018-0829-1.

39. Pozzi C, Andrulli S, Del Vecchio L, Melis P, Fogazzi GB, et al. Corticosteroid effectiveness in IgA nephropathy: long-term results of a randomized, controlled trial. J Am Soc Nephrol. 2004;15(1):157-63. https://doi.org/10.1 097/01.ASN.0000103869.08096.4F.

40. Rauen T, Eitner F, Fitzner C, Sommerer C, Zeier M, Otte B, et al. Intensive supportive care plus immunosuppression in IgA nephropathy. N Engl J Med. 2015;373(23):2225-36. https://doi.org/10.1056/NEJMoa1415463.

41. Zhou YH, Tang LG, Guo SL, Jin ZC, Wu MJ, Zang JJ, et al. Steroids in the treatment of IgA nephropathy to the improvement of renal survival: a systematic review and meta-analysis. PLoS One. 2011;6(4):e18788. https:// doi.org/10.1371/journal.pone.0018788.

42. Santisteban MM, Qi Y, Zubcevic J, Kim S, Yang T, Shenoy V, et al. Hypertension-linked pathophysiological alterations in the Gut. Circ Res. 2017:120(2):312-23. https://doi.org/10.1161/CIRCRESAHA.116.309006.

43. Hogg RJ, Lee J, Nardelli N, Julian BA, Cattran D, Waldo B, et al. Clinical trial to evaluate Omega-3 fatty acids and alternate day prednisone in patients with IgA nephropathy: report from the southwest pediatric nephrology study group. Clin J Am Soc Nephrol. 2006;1(3):467-74. https://doi.org/10.221 5/CJN.01020905.

44. Noriega BS, Sanchez-Gonzalez MA. Understanding the impact of Omega-3 rich diet on the Gut microbiota. Case Rep Med. 2016;2016:3089303.

45. Balfegó M, Canivell S, Hanzu FA, Sala-Vila A, Martínez-Medina M, Murillo S, et al. Effects of sardine-enriched diet on metabolic control, inflammation and gut microbiota in drug-naïve patients with type 2 diabetes: a pilot randomized trial. Lipids Health Dis. 2016;15(1):78. https://doi.org/10.1186/s12 944-016-0245-0.

46. Watson H, Mitra S, Croden FC, Taylor M. A randomised trial of the effect of omega-3 polyunsaturated fatty acid supplements on the human intestinal microbiota. Gut. 2018:67:1974-83.

47. Pu S, Khazanehei H, Jones PJ, Khafipour E. Interactions between obesity status and dietary intake of monounsaturated and polyunsaturated oils on human Gut microbiome profiles in the canola oil multicenter intervention trial (COMIT). Front Microbiol. 2016;7:1612

48. Rajkumar H, Mahmood N, Kumar M, Varikuti SR, Challa HR, et al. Effect of probiotic (VSL\#3) and omega-3 on lipid profile, insulin sensitivity, inflammatory markers, and gut colonization in overweight adults: a randomized, controlled trial. Mediat Inflamm. 2014:2014:348959.

49. Wei F, Xu H, Yan C, Rong C, Liu B, Zhou H. Changes of intestinal flora in patients with systemic lupus erythematosus in Northeast China. PLoS One. 2019;14(3):e0213063. https://doi.org/10.1371/journal.pone.0213063.
50. Larsen N, Vogensen FK, van den Berg FWJ, Nielsen DS, Andreasen AS, Pedersen BK, et al. Gut microbiota in human adults with type 2 diabetes differs from non-diabetic adults. PLoS One. 2010;5(2):e9085. https://doi.org/1 0.1371/journal.pone.0009085.

51. Haro C, Rangel-Zuniga OA, Alcala-Diaz JF, Gomez-Delgado F, Perez-Martinez $P$, et al. Intestinal microbiota is influenced by gender and body mass index. PLoS One. 2016;11:e0154090.

52. Nakayama J, Yamamoto A, Palermo-Conde LA, Higashi K, Sonomoto K, et al. Impact of Westernized Diet on Gut Microbiota in Children on Leyte Island. Front Microbiol. 2017;8:197.

53. De Filippo C, Cavalieri D, Di Paola M, Ramazzotti M, Poullet JB, et al. Impact of diet in shaping gut microbiota revealed by a comparative study in children from Europe and rural Africa. Proc Natl Acad Sci U S A. 2010; 107(33):14691-6. https://doi.org/10.1073/pnas.1005963107.

54. Tomova A, Bukovsky I, Rembert E, Yonas W, Alwarith J, Barnard ND, et al. The effects of vegetarian and vegan diets on Gut microbiota. Front Nutr. 2019;6:47-7. https://doi.org/10.3389/fnut.2019.00047.

55. Losasso C, Eckert EM, Mastrorilli E, Villiger J, Mancin M, et al. Assessing the influence of vegan, vegetarian and omnivore oriented westernized dietary styles on human Gut microbiota: a cross sectional study. Front Microbiol. 2018;9:317.

56. Salonen A, Lahti L, Salojärvi J, Holtrop G, Korpela K, Duncan SH, et al. Impact of diet and individual variation on intestinal microbiota composition and fermentation products in obese men. ISME J. 2014;8(11):2218-30. https:// doi.org/10.1038/ismej.2014.63.

57. Strauss J, Kaplan GG, Beck PL, Rioux K, Panaccione R, DeVinney R, et al. Invasive potential of gut mucosa-derived Fusobacterium nucleatum positively correlates with IBD status of the host. Inflamm Bowel Dis. 2011; 17(9):1971-8. https://doi.org/10.1002/ibd.21606.

58. Bullman S, Pedamallu CS, Sicinska E, Clancy TE, Zhang X, et al. Analysis of Fusobacterium persistence and antibiotic response in colorectal cancer. Science. 2017;358:1443-8.

59. McCoy AN, Araújo-Pérez F, Azcárate-Peril A, Yeh JJ, Sandler RS, et al. Fusobacterium is associated with colorectal adenomas. PLoS One. 2013;8(1): e53653. https://doi.org/10.1371/journal.pone.0053653.

60. McDonald D, Price MN, Goodrich J, Nawrocki EP, DeSantis TZ, et al. An improved Greengenes taxonomy with explicit ranks for ecological and evolutionary analyses of bacteria and archaea. ISME J. 2012;6(3):610-8. https://doi.org/10.1038/ismej.2011.139.

61. Lurie-Weinberger MN, Gophna U. Archaea in and on the human body: health implications and future directions. PLoS Pathog. 2015;11(6):e1004833. https://doi.org/10.1371/journal.ppat.1004833.

62. Chen $Y-Y$, Chen D-Q, Chen L, Liu J-R, Vaziri ND, Guo Y, et al. Microbiomemetabolome reveals the contribution of gut-kidney axis on kidney disease. J Transl Med. 2019;17(1):5. https://doi.org/10.1186/s12967-018-1756-4.

63. Schmitt R, Carlsson F, Mörgelin M, Tati R, Lindahl G, Karpman D. Tissue deposits of IgA-binding streptococcal M proteins in IgA nephropathy and Henoch-Schonlein purpura. Am J Pathol. 2010;176(2):608-18. https://doi. org/10.2353/ajpath.2010.090428.

64. Bernstein CN, Forbes JD. Gut microbiome in inflammatory bowel disease and other chronic immune-mediated inflammatory diseases. Inflamm Intestinal Dis. 2017;2(2):116-23. https://doi.org/10.1159/000481401.

65. Hevia A, Milani C, López P, Cuervo A, Arboleya S, et al. Intestinal Dysbiosis associated with systemic lupus Erythematosus. mBio. 2014;5:e01548-14.

66. Stilling RM, van de Wouw M, Clarke G, Stanton C, Dinan TG, Cryan JF. The neuropharmacology of butyrate: the bread and butter of the microbiotagut-brain axis? Neurochem Int. 2016;99:110-32. https://doi.org/10.1016/j. neuint.2016.06.011.

67. Hu X, Du J, Xie $Y$, Huang $Q$, Xiao $Y$, et al. Fecal microbiota characteristics of Chinese patients with primary IgA nephropathy: a cross-sectional study. BMC Nephrol. 2020;21(1):97. https://doi.org/10.1186/s12882-020-01741-9.

68. Wittebolle L, Marzorati M, Clement L, Balloi A, Daffonchio D, Heylen K, et al. Initial community evenness favours functionality under selective stress. Nature. 2009;458(7238):623-6. https://doi.org/10.1038/nature07840.

69. Ricotta C. A semantic taxonomy for diversity measures. Acta Biotheor. 2007; 55(1):23-33. https://doi.org/10.1007/s10441-007-9008-7.

\section{Publisher's Note}

Springer Nature remains neutral with regard to jurisdictional claims in published maps and institutional affiliations. 